\section{Double staining of elastic fibre and immunohistochemistry is helpful to differentiate pleural invasion of lung cancer}

Visceral pleural invasion (VPI) is one of the critical determining factors for pathological stage of lung cancer. The pleural invasion (PL) status can be divided into four categories: PL0, PL1, PL2 and PL3, depending on the spatial relationship between the tumour tissue and outer elastic layer of pleura, visceral pleura surface and parietal pleura, respectively. Elastic stains are recommended if it is difficult to distinguish PL status by H\&E staining. As reported, 19 of 100 lung cancer specimens were redefined as PL1 which were formerly deemed as PLO by HE stain only, ${ }^{1}$ changing the stage from T1 (stage IA) to T2 (stage IB), which is essential for chemotherapy after surgery. ${ }^{2}$ Nonetheless, even assisted by elastic stains, VPI assessment is still difficult in some scenarios marked by multiple layers of elastic fibres, inflammatory infiltrates, fibrotic proliferation or carbon deposition which makes the location of scattered tumour cells barely discernible. To solve this technical problem, we developed a double staining method to show the spatial relationship between tumour cells and elastic fibres. Specifically speaking, the elastic fibres and tumour cells are stained and displayed by different colours on one slide, to determine whether the pleura is invaded.

Double staining process: FormalinFixed, Paraffin-Embedded (FFPE) blocks were sectioned by thickness 3-5 um for double staining. Step one is routine immunohistochemistry staining, including: (1) antigen retrieval for $23 \mathrm{~min}$ in $97^{\circ} \mathrm{C},(2)$ washing with distilled water, (3) 3\% hydrogen peroxide solution incubated for $10 \mathrm{~min}$ to remove endogenous peroxidase, (4) rinsing with phosphate balanced solution (PBS) $3 \mathrm{~min}$ for three times, (5) add primary antibody (thyriod transcription factor-1 (TTF-1), and PanCK (AE1/AE3), (Zhongshan, Beijing, China)) and incubate at room temperature for 40-60 $\mathrm{min}$, (6) rinsing with PBS, add the secondary antibody (Horseradish Peroxidase (HRP)) and incubate for 10-15 $\mathrm{min}$ at room temperature, (7) rinsing with PBS $3 \mathrm{~min}$ for three times and then diaminobenzidine (DAB) colour development. Second step is double dyeing of elastic fibre
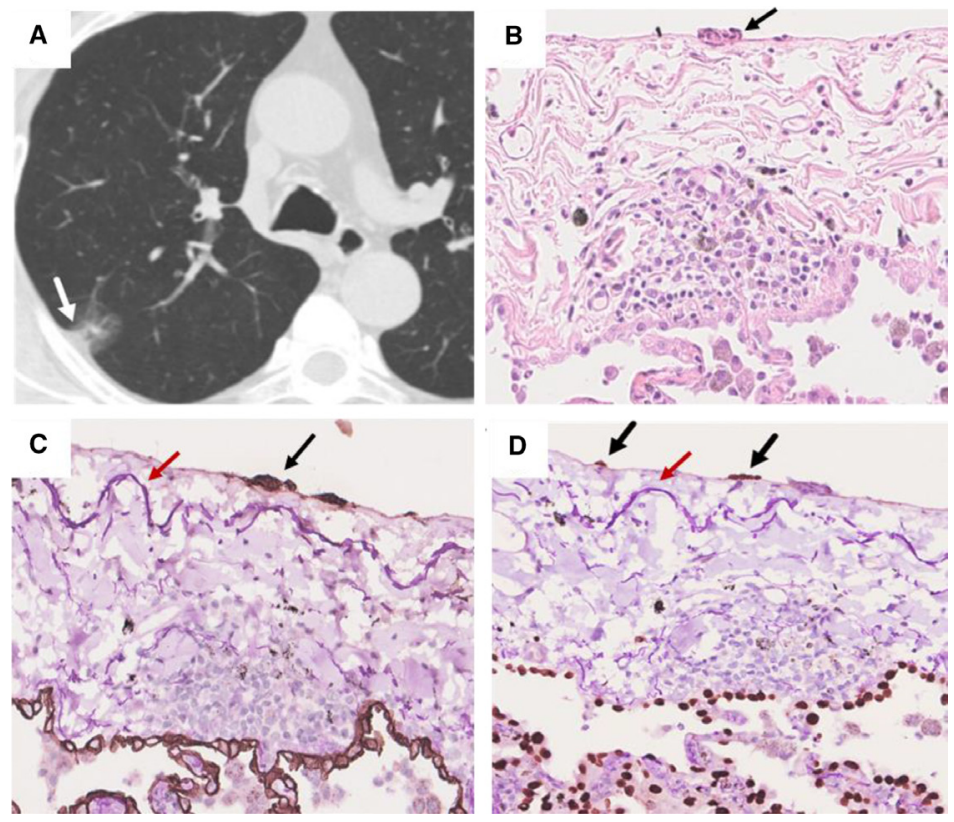

Figure 1 (A) Representative case of double staining. (A) CT scan shows a part-solid nodule with the entire size of $22.9 \times 19.5 \mathrm{~mm}$ and the maximum diameter of the solid component of $9.2 \mathrm{~mm}$ adjacent with pleura (white arrow). (B) H\&E image shows several large hyperchromatic cells on the surface of pleura (black arrows, $\times 200$ ), which confirms further by elasticimmunohistochemistry double staining of AE1/AE3 (C, black arrows) and TTF-1 (D, black arrows), with the outer elastic fibre stained with purple(red arrow, $\times 200)$. TTF-1, thyriod transcription factor-1. using a Fuxi Assay (Fuxi Bio, Guiyang, China) according to the instruction for $10-15 \mathrm{~min}$, then $70 \%$ ethanol is used for counterstaining with haematoxylin. Finally, dehydrate and transparently seal after washing.

TTF-1 or AE1/AE3 shows a dark brown immunohistochemistry (IHC) signal, with the former exclusively nuclear stained and the latter localised to the cytoplasm. Elastic fibres are stained in purple while all the nuclei are stained blue with haematoxylin, including tumour cells, stromal cells, lymphocytes and so on. Figure 1 shows a nodule scanned in CT with slight pleural stretch, which is hard to discern in routine $\mathrm{H} \& \mathrm{E}$ slides since the elastic fibres are intact stained in an ambiguous pink architecture, but with the help of double staining, we can easily recognise tumour cells attached on the surface of pleura to a correct diagnosis for PL2. Figure 2 shows
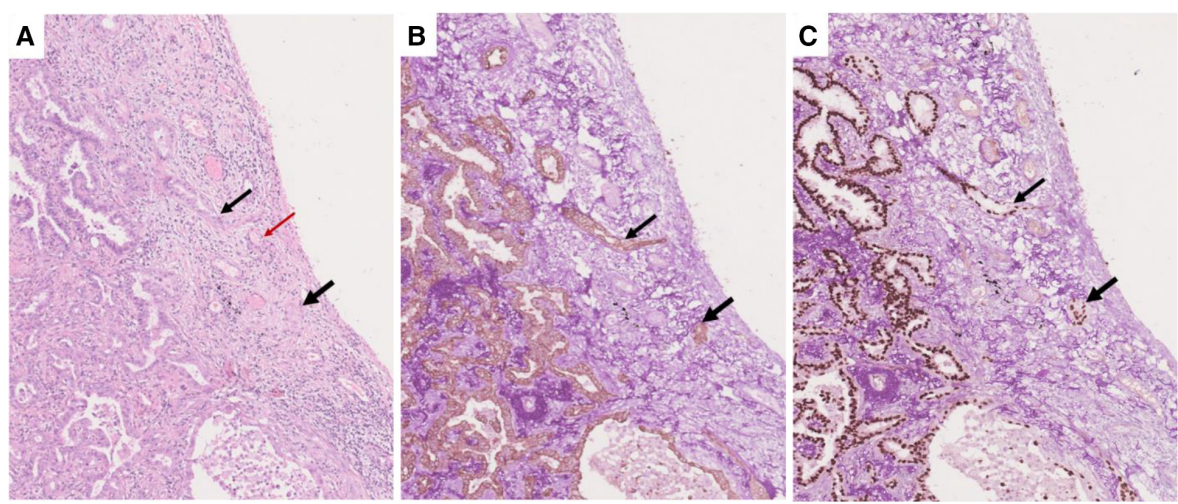

Figure 2 Another difficult case for VPI status. (A) H\&E staining shows the pleura full of fibres and capillaries (red arrow) with an acinar structure protruding towards the surface of pleura (thin black arrow, $\times 100)$. Double staining of AE1/AE3 (B, dark brown in cytoplasm), TTF-1 (C, dark brown in nucleus) and elastic fibre (in purple, $\times 100$ ) confirms the protruding acinar structure to be adenocarcinoma (thin dark arrow from $A$ to $C$ ) and further confirmed a capillary-like adenocarcinoma area (bold dark arrow from $A$ to $C$ ) so as to achieve a correct diagnosis of PL1. PL, pleural invasion; TTF-1, thyriod transcription factor-1; VPI, visceral pleural invasion. 
a more difficult case, from the H\&E slide, we can hardly recognise elastic fibre and cancer cells from the conspicuous hyperplastic fibres and capillaries. However, our double staining method can easily identify the location of adenocarcinoma cells and elastic fibres and screen out the proliferative capillaries, which are obviously similar to acinar adenocarcinoma on $\mathrm{H} \& \mathrm{E}$ section.

Compared with the traditional elastic fibre staining, our double staining method has the following advantages: (1) easy interpretation, high repeatability and less controversy. (2) Working time is shorter for $2 / 3$ than that of immunohistochemical staining and elastic fibre staining alone (2.5 hours vs 6 hours). (3) The cost is small. Our method is worth popularising for replacing routine elastic fibre staining to make a more correct diagnosis for pleural invasion.

Guihua Shen, ${ }^{1}$ Jiyan Dong $\odot{ }^{2}$ Zheng Xiang, ${ }^{3}$ Li Liu, ${ }^{2}$ Lin Yang ${ }^{2}$

'Department of Pathology, National Cancer Center/ National Clinical Research Center for Cancer/Cancer Hospital/Shenzhen Hospital, Chinese Academy of Medical Sciences and Peking Union Medical College, Shenzhen, China

${ }^{2}$ Department of Pathology, National Cancer Center/ National Clinical Research Center for Cancer/Cancer Hospital, Chinese Academy of Medical Sciences and Peking Union Medical College, Beijing, China
${ }^{3}$ Fuxi Biological Technology Co., Ltd, Guizhou, China

Correspondence to Dr Lin Yang, Department of Pathology, National Cancer Center/National Clinical Research Center for Cancer/Cancer Hospital, Chinese Academy of Medical Sciences and Peking Union Medical College, Peking Union Medical College Hospital, Chaoyang-qu, Beijing, China; linyang0616@ 126.com

\section{Handling editor Runjan Chetty.}

Contributors GS: investigation, roles/writing original draft. JD: formal analysis, writing review and editing. ZX: methodology, software. LL: project administration, data curation. LY: conceptualisation, funding acquisition, writing review and editing, supervision.

Funding The work is funded by Sanming Project of Medicine in Shenzhen (Grant No.SZSM201812076).

Competing interests None declared.

Patient consent for publication Not applicable.

Ethics approval The study was approved by the ethics committee and institutional review board of Cancer Hospital, Chinese Academy of Medical Sciences (CHCAMS), and all patients were exempted for informed consent.

Provenance and peer review Not commissioned; internally peer reviewed.

\section{(2) OPEN ACCESS}

Open access This is an open access article distributed in accordance with the Creative Commons Attribution Non Commercial (CC BY-NC 4.0) license, which permits others to distribute, remix, adapt, build upon this work non-commercially, and license their derivative works on different terms, provided the original work is properly cited, appropriate credit is given, any changes made indicated, and the use is non-commercial. See: http://creativecommons.org/ licenses/by-nc/4.01.

(C) Author(s) (or their employer(s)) 2021. Re-use permitted under CC BY-NC. No commercial re-use. See rights and permissions. Published by BMJ.

GS and JD are joint first authors.

$$
\text { A Check for updates }
$$

To cite Shen G, Dong J, Xiang Z, et al. J Clin Pathol Epub ahead of print: [please include Day Month Year]. doi:10.1136/jclinpath-2021-207779

Received 24 June 2021

Accepted 25 July 2021

J Clin Pathol 2021;0:1-2.

doi:10.1136/jclinpath-2021-207779

\section{ORCID iD}

Jiyan Dong http://orcid.org/0000-0002-1316-216X

\section{REFERENCES}

1 Taube JM, Askin FB, Brock MV, et al. Impact of elastic staining on the staging of peripheral lung cancers. Am J Surg Pathol 2007;31:953-6.

2 Goldstraw P, Chansky K, Crowley J, et al. The IASLC lung cancer staging project: proposals for revision of the TNM stage groupings in the forthcoming (eighth) edition of the TNM classification for lung cancer. J Thorac Oncol 2016:11:39-51. 